\title{
The Effects of Retail Ambient Music and Noise on Food Purchases: An Abstract
}

\author{
Dipayan Biswas, Kaisa Lund, and Courtney Szocs
}

\begin{abstract}
Ambient sound, which includes both ambient music and noise, is present in nearly all retail stores and restaurants. Yet, there is great variation in the volume of this ambient sound. Specifically, some restaurants have very high levels of sound and others have very low levels of sound. This research examines how the volume of ambient music and noise influences consumers' food choices. A series of field and laboratory studies show that low (vs. high)-volume ambient music and noise lead to healthier food choices. Process evidence suggests that the effects of ambient sound on food choices are driven by greater relaxation induced with low-volume ambient sound. The findings of this research suggest that restaurant, cafeteria, and supermarket managers wanting to nudge healthier food choices might simply need to adjust the volume on ambient sound systems.
\end{abstract}

\author{
D. Biswas \\ University of South Florida, Tampa, FL, USA \\ e-mail: dbiswas@usf.edu \\ K. Lund \\ Linneaus University, Växjö, Sweden \\ e-mail: kaisa.lund@lnu.edu \\ C. Szocs $(\bowtie)$ \\ Portland State University, Portland, OR, USA \\ e-mail: courtne@pdx.edu
}

